\title{
Social data analysis instruction and the MISSIS system
}

\author{
RONALD E. ANDERSON, KARL R. KROHN, and MARK L. MONGIAT \\ University of Minnesota, Minneapolis, Minnesota 55545
}

\begin{abstract}
Social data analysis instruction is an enterprise providing experiences to develop problem solving skills. A proper focus upon analysis can best be provided with a data analysis package stressing simplicity rather than complexity. MISSIS is such a system, and it is designed to operate on small computers. Small technology can provide a training ground for making sound decisions in analyzing data.
\end{abstract}

\section{INSTRUCTIONAL CONSIDERATIONS}

Most discussions of statistical computing techniques for instruction focus upon the teaching of statistics rather than upon analysis. Our pedagogical concern is analysis, specifically, the analysis of social data such as surveys.

Social data analysis instruction should be seen as an enterprise providing experiences for students to develop problem solving skills. The "problem," more aptly called the "challenge," is to find and communicate evidence of meaningful data patterns or regularities that bear upon substantive hypotheses. The analysis process begins with inquiry or the formulation of useful questions; it culminates with the interpretation of results. To perform data analysis, then, implies the completion of a total or long-term process involving problem formulation, data management, data processing, and data interpretation.

This problem solving perspective can be rephrased in terms of instructional objectives. Specifically, we propose three objectives: (1) inquiry, which consists primarily of asking the most useful questions, (2) productivity, a term used to denote successful problem solving, and (3) literacy to implement successful problem solving strategies. With regard to the last objective, the most crucial skills are statistical literacy and computer literacy. In order to be productive in contemporary research environments, one must be facile in these functional arenas.

It is difficult, if not impossible, to achieve these instructional objectives without a research apprenticeship environment where the student has facilities and feedback or guidance. Such an environment should have a diversity of data sets, an assortment of computer software packages, and the necessary computing machinery for utilizing these resources. Such diversity is essential to the training enterprise because it is necessary to progressively give students more and more freedom to select among techniques and strategies for analyzing different types of data.

While a rich technical environment is important, constraints must be placed on technological complexity. Techniques can be designed to be simple and easy to use. There are numerous computer packages designed to do social data analysis, but each has inherent weaknesses as well as strengths. One key problem is that as packages like SPSS, SAS, and BMDP evolve, they become cumbersome and difficult to use, ironically, in the interest of flexibility and variety. The major design consideration in our effort is to return to simplicity, which is to say, small technology. Our goals are to make relatively small demands for student expertise and to make the software work on small hardware, for example, microcomputers and minicomputers. Obviously, losses accrue from reductions of scale, but "big" is not better if complexity or cost inhibits access to resources. We seek simplification whenever productivity is not dramatically curtailed.

\section{THE MISSIS SYSTEM: AN OVERVIEW}

MISSIS (Minnesota interactive statistical system for instruction in sociology) is an integrated system for the analysis of social science data. MISSIS was developed by the sociology department of the University of Minnesota, Minneapolis, Minnesota. It was designed to maximize both ease of use and flexibility of analysis, in order to serve the novice user as well as the experienced researcher. While MISSIS was specifically developed for instruction in sociology, its application potential extends to other fields as well.

As an integrated and interactive system, MISSIS permits analysis of a data set in a stepwise manner, viewing the results of one analysis procedure before deciding upon the selection of a subsequent statistical analysis. The rapid feedback of the system speeds the learning process and makes the task less stressful.

MISSIS is an outgrowth of an earlier system, MISS, which was written in FOCAL for a PDP-8L minicomputer (Anderson \& Gross, 1972; Anderson, Krohn, \& Mongiat, 1974; Cleary, 1973). MISSIS borrows much of the logic, syntax, and documentation from the earlier system. It is, however, completely rewritten in 
FORTRAN IV and is currently installed under the KRONOS and NOS operating systems on the Control Data CYBER series computers. It also operates on a Digital Equipment PDP.10. The large computer environment has allowed for the expansion of some capabilities, but the basic structure of the original MISS system has been preserved. A user's manual titled MISSIS Primer is available from MECC. ${ }^{1}$

The existing version of MISSIS, like MINITAB and many other statistical packages, is designed to have the entire working data array stored in central memory. While this improves speed, it prohibits processing of very large data files. At present, the work space for data is limited to 5,000 elements, for example, 10 variables and 500 observations (cases). The micro version of MISSIS, which is under development, will work from data files on floppy disks or diskettes. While this is slower, it offers an important advantage for instruction because students can maintain copies of data on their own inexpensive disks.

As shown in Figure 1, MISSIS assumes that data will be in either observational (raw) or reduced form. The reduced data arrays can be either bivariate tabulations or a matrix of correlation coefficients. Once the data type and the source of the data have been defined to the system by the user, MISSIS proceeds to wait for a data action request. The student user is given a menu of data action alternatives in much the same way as the UCSD PASCAL operating system communicates with the user. The user continues to select data processing (including analysis) options until he or she either wants a new data array or is ready to quit.

The list of action codes is explained in Figure 2. If the user chooses " $A$ " for analysis, then he or she is immediately asked to select a specific analysis procedure. Using the terminology of Sonquist and Dunkelberg (1977), MISSIS provides for data entry with the new data command, data editing with the change and list commands, data transformation with the recode and transformation commands, and data definition with the missing data command. The action code list also contains options to create a permanent data file on disk and to get "help" in the form of instant documentation.

An illustrative session with MISSIS is displayed in Figure 3 where the user first answers a series of questions about the organization and source of the data before entering a small data array. As shown in the figure, an action code list is immediately displayed. In the Figure 3 session, the user selected the option to change or correct the data. Such data management tasks are made very simple, asking the user to merely answer a few questions. The same is true of the recoding operation, which is demonstrated in Figure 4. This dialogue approach makes it possible for someone with essentially no previous experience to complete such tasks successfully. If a command language approach, in the spirit of SPSS, SAS, and so on, were used, it would add flexibility but would substantially increase the requisite expertise demanded of users.

Missing data declaration is a major concern in most social research. Many data analysis packages demand that the user recode the missing data values to some special value that the package treats as missing. The major alternative approach to definition of missing values is to retain a limited set of missing values for any given attribute (variable). Like SPSS, MISSIS allows the user to define up to three missing values for each variable. If one does not declare missing values for a variable, then a " 0 " (zero) is assumed to be a missing value by default. It is possible to override this default, as shown in Figure 5. In this illustration, the entire data array is listed first to show the nature of the values. Then, after requesting the missing data action, the user defines two missing values for each of two variables. Following this definition activity, the program prints a missing data table for the user's record. Any observations with missing values as defined in the missing values table are subsequently dropped from any analysis.

\section{INSTRUCTIONAL USE OF MISSIS}

The availability of programmed facilities such as transformation and missing data declaration make it possible for students to learn how to carry out the entire process of social data processing. Assignments can be designed to encourage such learning in a relatively short time frame. For instance, students are given the task of performing a statistical test (e.g., a t test) on the set of data listed in Figure 5. A code book describing the data is provided, but the students are not explicitly told to define missing data. If they forget to define missing values, and many do forget, the results are drastically inaccurate because the missing values are so far out of range. Seeing such discrepant results tends to quickly teach a great deal of caution.

As stated earlier, it is desirable to have a variety of analysis capabilities available for student choices. Figure 6 lists the existing analysis procedures available in MISSIS. Not only is there variety in this set, but it is not very difficult to add an additional procedure to the set. Figures 7 and 8 illustrate some of the analysis procedures. Figure 7 shows the analysis of a crosstabulation table. The rows and columns are labeled " 0 " because the table was entered as data. Note that the statistical measures are grouped into a nominal and an ordinal set. This grouping allows the teacher to directly assess student understanding of the way in which measurement level is directly related to choice of coefficient.

A diversity of data sets as well as a diversity of analytical techniques is also important for a full instructional environment. For our research and statistics courses, we have begun to accumulate a number of 


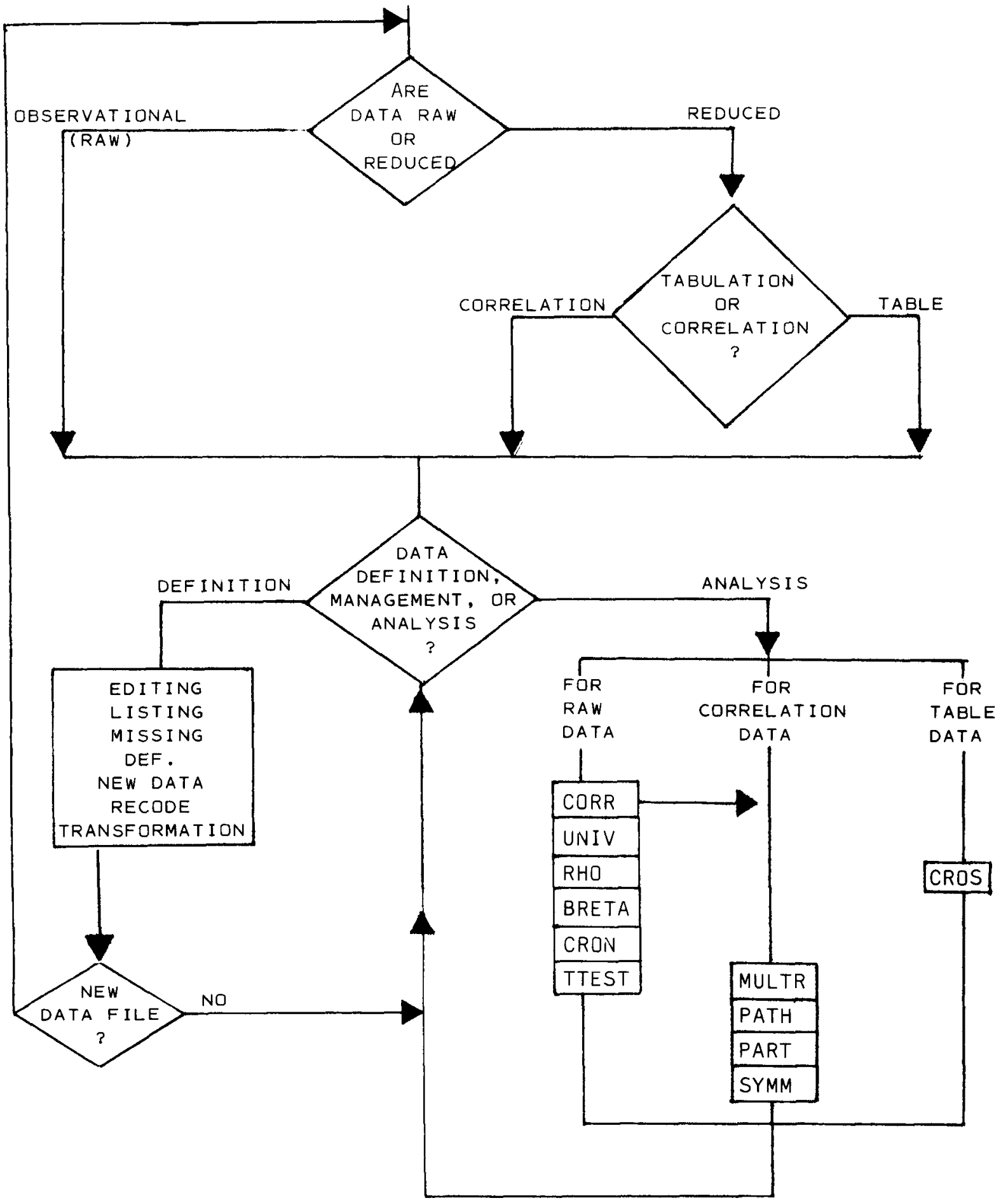

Figure 1.

small data sets. To illustrate: (1) CAS12 is a 214-student survey of attitudes toward computerization. Several different structural and social psychological variables make it possible to perform a wide assortment of data processing tasks, as well as multivariate analyses.
(2) CARTROC is the name of a file obtained from Namboodiri, Carter, \& Blalock (1975). It is a simulationgenerated data file designed to illustrate a number of unusual as well as typical patterns of interrelationships. If the instructor is familiar with the data patterns, it 
A ANALYSIS --used to request the execution of a particular analysis procedure. After responding "A", MISSIS will ask for the procedure name.

C CHANGE

--used to change or append data. If errors have been made during data entry and they were not corrected before the carriage return, corrections may be made by typing " $C$ " when MISSIS gives the ACTION command. This option may also be used if you wish to append (add) data to the end of the dataset.

$H$ HELP --the "H" response will cause a listing of the ACTION command options to be printed.

L LIST

--the "L" option will allow you to request that your dataset be listed. MISSIS will list either the entire dataset or a subset of cases (rows) upon your request. It's a good idea to list the data following data entry to check for errors.

M MISSING DATA --all of the analysis procedures of MISSIS allow for the handling of missing data. Missing data are excluded from the analysis (1ist-wise deletion). Missing Data Definition is used to declare missing values for your variables. If not used, zeros will be treated as missing by defauTt.

$N$ NEW DATA --at anytime during the computing session, when provided with an ACTION command, you may initiate the entry of a new dataset by responding "N". MISSIS will respond by asking for the source of the new data (TTY,DISK).

$P$ PERMANENT --a dataset entered from the keyboard may be saved as a permanent disk file by responding "P" to the ACTION command. This will allow you to use the data at a later time in the same computing session (by requesting NEW DATA), or at a later session.

$R$ RECODE --from time-to-time it may be desirable to change or group the values assigned to response categories. This capability is provided by the "R" response to the ACTION command.

S STOP

--the "S" response is used to terminate MISSIS. Following the "S" response, type "BYE" to log-off the computer.

T IRANSFORMATION--this option allows for the replacement of the coded values of one or more variables with the results of some mathematical transformation on those values.

Figure 2. Action code list. 
-MISSIS

WELCOME TO MISSIS.

INSTRUCTIONS (YES OR NO)

? NO

DATA TYPE (RAW, MATR IX)

? MATRIX

DATA SOURCE (TTY, DISK)

$?$ TTY

IS MATRIX CORRELATION(CORR) OR TABULATION(TABL)

? TABL

TELETYPE INPUT FOR TABULATION MATRIX.

ENTER NO. OF COLS. IN MATRIX.

? 3

ENTER NO. OF ROWS IN MATRIX.

? 3

TYPE IN TABULATION MATRIX.

SEPARATE EACH ENTRY WITH EITHER A

SPACE OR A COMMA.

ENTER DATA, ONE CASE(ROW) AT A TIME.

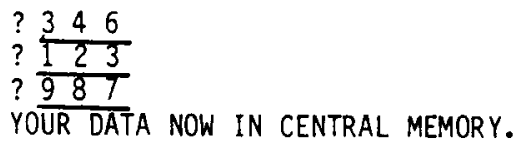

ACTION (A,C, H, L,M N N P, R, S,T)

$? \underline{\mathrm{C}}$

DATA CHANGE(C) OR APPEND(A)

$? \mathrm{C}$

DATA CHANGE

WHICH ROW

? 1

WHICH COLUMN

? 3

WHAT IS THE CORRECT VALUE

? 5

CORRECTED RECORD

$\begin{array}{llll}1 & 3.000 & 4.000 & 5.000\end{array}$

ANY OTHER CHANGES (YES, NO)

? YES

DATA CHANGE(C) OR APPEND(A)

? A

DATA APPEND

HOW MANY ROWS ARE YOU GOING TO APPEND

? 1

TYPE IN THE APPENDED DATA. BE SURE TO

SEPARATE EACH ENTRY WITH EITHER A SPACE OR COMMA. ? 346

Figure 3.

can be a very effective teaching tool. (3) SEXUALITY is a correlation matrix taken from a recent research report analyzing data taken from the national General Social Survey. Various models can be tested for the purpose of analyzing factors underlying acceptance of extramarital and premarital sexual activiey. (4) LYNCHING is another correlation matrix but based upon demographic data on 50 counties. The basic question to explore in this data set is whether or not the rate of lynching at the turn of the century was associated with political and social conditions. (5) ADOPTION is still another correlation matrix from a recently published study of factors underlying high school teacher adoption of instructional computer technology. The data are especially useful to test path models of adoption or innovation. The path analysis procedure (see Figure 8 ) makes it rather simple to generate the statistics necessary to test different models.

These exemplary data arrays, while interesting, are all very small and do not do full justice to the capacity of the MISSIS system. Most important, a large number of data sets such as these can be maintained on-line without a lot of effort or cost to support the learning environment.

\section{DESIGN DECISIONS}

While some design issues such as size of data and style of missing data declaration have been discussed, there are several other major considerations in the design of systems such as MISSIS. One is the manner in which the system interfaces with other systems. The current version of MISSIS has the ability to open and close files but otherwise is not intimately interconnected with the operating system. For instance, to use system programs or to execute another package, one must exit from MISSIS and run another program. Incidentally, MISSIS users are encouraged to use other packages such

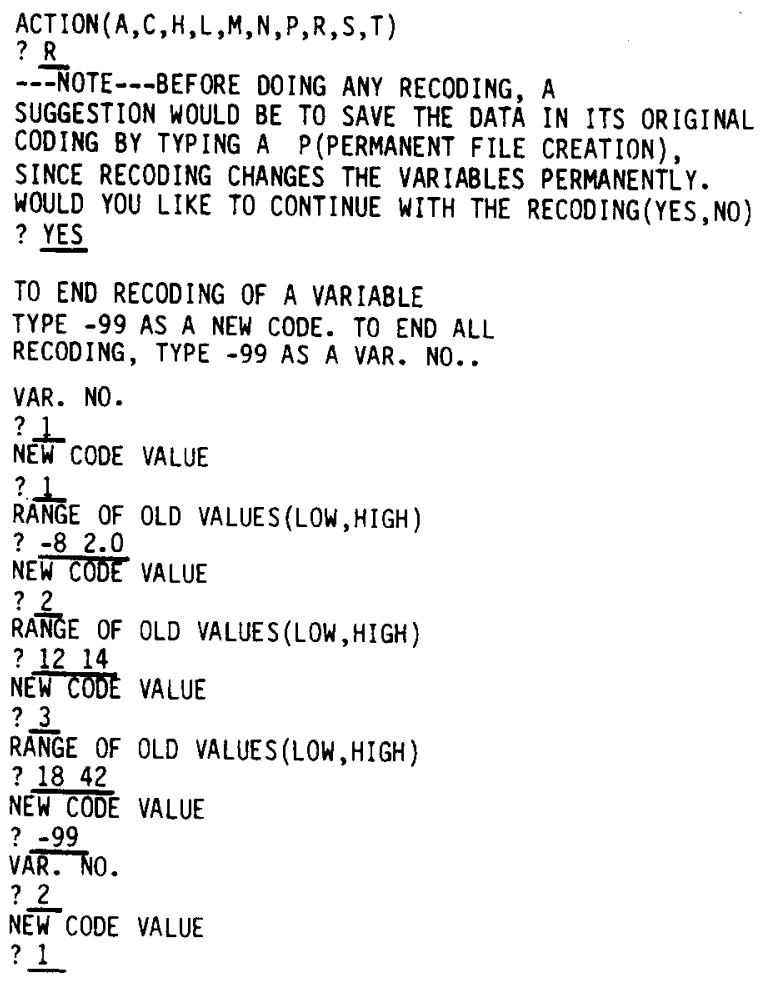

Figure 4. 
$A C T I O N(A, C, H, L, M, N, P, R, S, T)$

$? \underline{L}$

DATA LISTING OF HOW MANY CASES

$? \underline{10}$

VAR.

$$
2
$$

$\begin{array}{rrr}1 & 2.000 & 999.000 \\ 2 & 2.000 & 1.000 \\ 3 & 999.000 & 3.000 \\ 4 & 1.000 & 1.000 \\ 5 & 2.100 & 2.000 \\ 6 & 2.000 & 998.000 \\ 7 & 3.000 & 2.000 \\ 8 & 998.000 & 2.000 \\ 9 & 3.000 & 1.000 \\ 10 & 1.000 & 3.000\end{array}$

$\operatorname{ACTION}(A, C, H, L, M, N, P, R, S, T)$

? M

MISSING DATA DEFINITION

ENTER VAR. NO., FOLLOWED BY 1 TO 3 MISSING DATA CODES. (SEPARATED BY EITHER A SPACE OR COMMA)

TO EXIT, TYPE A CARRIAGE RETURN.

ENTER DATA, ONE CASE (ROW) AT A TIME.
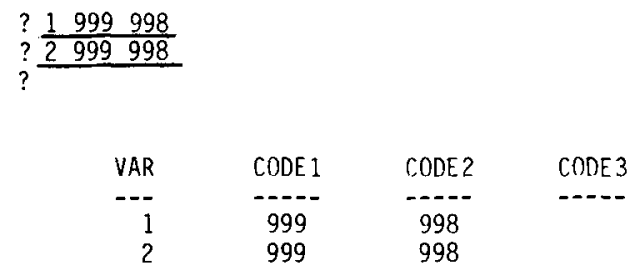

$A C T I O N(A, C, H, L, M, N, P, R, S, T)$

Figure 5. Defining missing data.

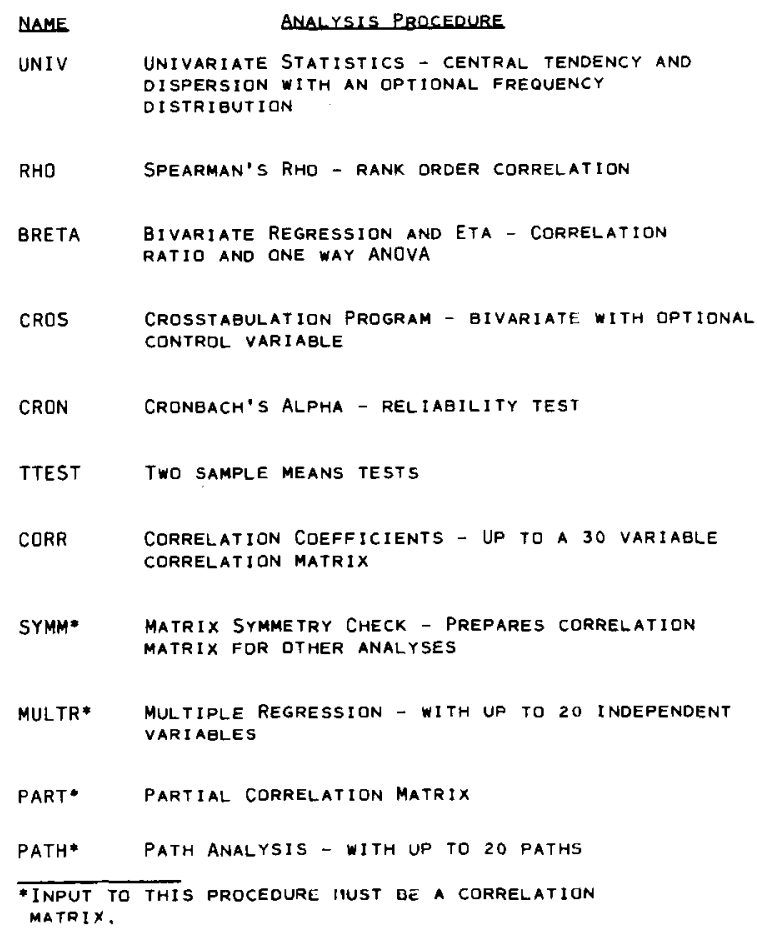

Figure 6. List of analysis procedures.

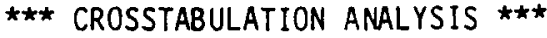

DO YOU WANT NOMINAL STATISTICS(YES, NO)

? YES

DO YOU WANT ORDINAL STATISTICS(YES, NO)

? YES

TOTAL

$$
\begin{array}{rrrrrr}
\text { CODE } & 0 & 0 & 0 & 0 & \\
& & & & & \\
0 & 12 & 5 & 6 & 8 & 31 \\
& 52.2 & 27.8 & 60.0 & 40.0 & 43.7 \\
0 & 11 & 13 & 4 & - & 12 \\
& 47.8 & 72.2 & 40.0 & 60.0 & 56.3
\end{array}
$$

\begin{tabular}{|c|c|c|c|}
\hline $\begin{array}{l}\text { PEARSON'S CONTINGEN } \\
\text { CRAMER'S } V= \\
\text { GOODMAN-KRUSKAL TAU } \\
\text { GOODMAN-KRUSKAL TAU } \\
\text { LAMBDA }(Y . X)= \\
\text { LAMBDA }(X . Y)= \\
\text { LAMBDA }(S Y M M E T R I C)\end{array}$ & $\begin{array}{l}\text { ICY COEFF } \\
88 \\
(Y . X)= \\
(X . Y)= \\
0968 \\
0417 \\
=\quad .06\end{array}$ & $\begin{array}{l}\text { IENT }= \\
.0524 \\
.0168\end{array}$ & .2231 \\
\hline $\begin{array}{l}\text { GAMMA }= \\
\text { KENDALL'S TAU A }= \\
\text { KENDALL'S TAU } B= \\
\text { KENDALL'S TAU C }= \\
\text { SOMER'S D }(Y . X)= \\
\text { SOMER'S D }(X . Y)= \\
\text { SOMER'S D (SYMMETRI }\end{array}$ & $\begin{array}{l}\text { SIGNIF } \\
.0310 \\
.0509 \\
.0611 \\
.0418 \\
.0621 \\
.=\end{array}$ & ICE: $Z$ & .302 \\
\hline
\end{tabular}

$\begin{array}{lrrrrr}\text { TOTAL } & 23 & 18 & 10 & 20 & 71\end{array}$ 100.0100 .0100 .0100 .0

Figure 7. Cross-tabulation analysis.

as MINITAB that are interactive and contain capabilities not found in MISSIS. Future versions of MISSIS, for example, a USCD PASCAL version of MISSIS, will maintain an even closer interface with the operating system for purposes of data management.

Another important concern is the range of output devices supported. Presently, MISSIS is oriented toward printing keyboard terminals, in that the output is not paged for screen viewing. A microcomputer version of MISSIS will necessarily support both types of interactive output devices. If we attempt to support MISSIS on an APPLE II microcomputer, we will have to provide for narrow (e.g., 40-character) screen widths. In addition, many of the analysis procedures will be modified to take advantage of graphics capabilities and other special features such as color.

Another design issue is the extent to which data definition facilities are provided. As already stated, missing data definition is critical. Other features such as labeling of variables and variable values are not essential 


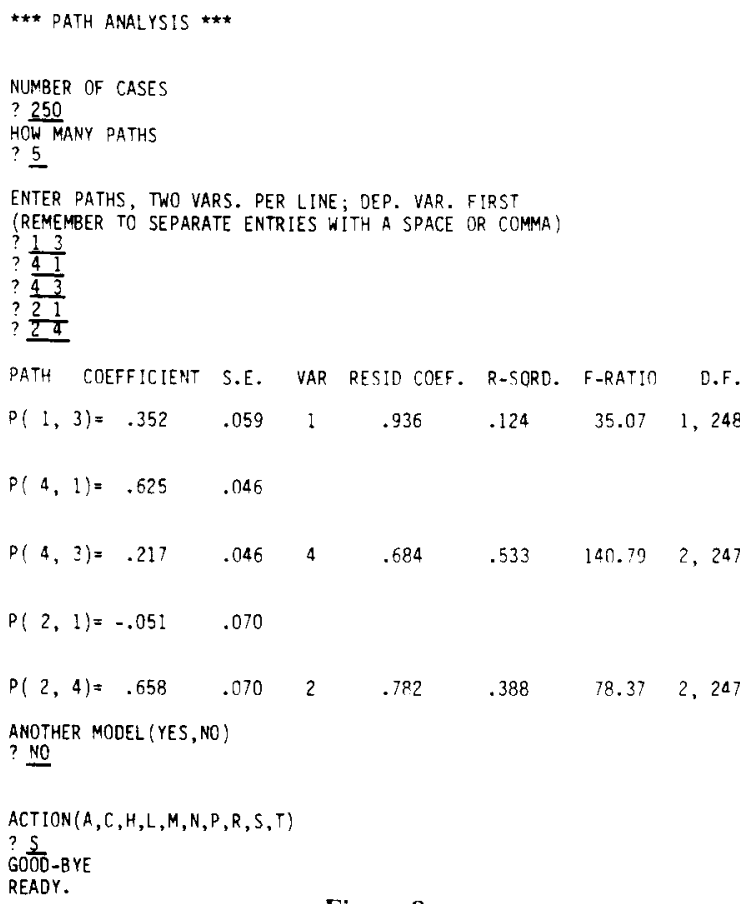

Figure 8.

and can be sacrificed in the interest of simplicity. MISSIS presently does not provide for format definition of the input data because the input data are assumed to be freefield with spaces between values. Future versions of MISSIS will be more oriented toward the hybrid interpreter-compiler system outlined by Coover, Dyer, Gross, Johnson, Lutgen, and Miner (1975). Consequently, many of the limitations we have been discussing might be easily overridden by insertion of interpretive statements into the package procedures.

\section{SUMMARY}

Producing effective software to challenge students to learn social data analysis is in itself a difficult challenge. A most critical consideration is the tradeoff between simplicity and power or flexibility. MISSIS demonstrates that it is possible to build a system that does not drastically sacrifice flexibility in the pursuit of simplicity. New technology (i.e., microcomputers and graphics capabilities) makes it even more feasible to achieve our goals.

\section{REFERENCES}

Anderson, R. E., \& Gross, J. Minicomputers in a social science instructional context. Proceedings of the ACM Conference. New York: Association for Computing Machinery, 1972.

Anderson, R. E., Krohn, K., \& Mongiat, M. L. The Minnesota interactive statistical system. Behavior Research Methods \& Instrumentation, 1974, 6, 194-200.

Cle ary, J. D. MISS primer. Minneapolis, Minn: Sociology Data Center, Department of Sociology, University of Minnesota, 1973.

Coover, E. R., Dyer, J. A., Gross, J. R., Johnson, C. C., Lutgen, G. L., \& Miner, J. F. Design of an optimally compatible social data analysis system: The first steps. Social Science Information, 1975, 13, 105-146.

Namboodiri, N. K., Carter, L. F., \& Blalock, H. M., Jr. Applied multivariate analysis and experimental design. New York: McGraw-Hill, 1975.

Sonquist, J. A., \& Dunkelberg, W. C. Survey data processing handbook. Englewood Cliffs, N.J: Prentice-Hall, 1977.

\section{NOTE}

1. The MISSIS Primer can be obtained from MECC, 2520 Broadway Drive, St. Paul, Minnesota 55113 for $\$ 3.50$. Copies of the software are available from the authors. 